\title{
Neutron-induced cross sections of actinides via the surrogate-reaction method
}

Q. Ducasse ${ }^{1,6}$, B. Jurado ${ }^{1}$, M. Aiche ${ }^{1}$, L. Mathieu ${ }^{1}$, T. Tornyi ${ }^{2}$, A. Görgen ${ }^{2}$, J. N. Wilson ${ }^{3}$, G. Barreau ${ }^{1}$, I. Companis ${ }^{1}$, S. Czajkowski ${ }^{1}$, F. Giacoppo ${ }^{2}$, F. Gunsing ${ }^{4}$, M. Guttormsen ${ }^{2}$, A. C. Larsen ${ }^{2}$, M. Lebois ${ }^{3}$, J. Matarranz ${ }^{1}$, T. Renstrøm ${ }^{2}$, S. Rose ${ }^{2}$, S. Siem ${ }^{2}$, I. Tsekhanovich ${ }^{1}$, G. M. Tveten ${ }^{2}$, T. W. Hagen ${ }^{2}$, M. Wiedeking, O. Serot ${ }^{6}$, G. Boutoux ${ }^{7}$, P. Chau ${ }^{7}$, V. Méot ${ }^{7}$, O. Roig $^{7}$

${ }^{1}$ CENBG, Chemin du Solarium B.P. 120, 33175 Gradignan, France

${ }^{2}$ University of Oslo, Department of Physics, P.O. Box 1048, Blindern 0316 Oslo, Norway

${ }^{3}$ IPN Orsay, 15 rue G. Clémenceau, 91406 Orsay cedex, France

${ }^{4}$ CEA Saclay, DSM/Irfu, 91191 Gif-sur-Yvette cedex, France

5 iThemba LABS, P.O. Box 722, 7129 Somerset West, South Africa

${ }^{6}$ CEA Cadarache, DEN/DER/SPRC/LEPh, 13108 Saint Paul lez Durance, France

${ }^{7}$ CEA DAM DIF, 91297 Arpajon, France

\begin{abstract}
The surrogate-reaction method is an indirect way of determining cross sections for reactions that proceed through a compound nucleus. This technique may enable neutron-induced cross sections to be extracted for short-lived nuclei that otherwise cannot be measured. However, the validity of the surrogate method for extracting capture cross sections has to be investigated. In this work we study the reactions ${ }^{238} \mathrm{U}(\mathrm{d}, \mathrm{p}){ }^{239} \mathrm{U},{ }^{238} \mathrm{U}\left({ }^{3} \mathrm{He}, \mathrm{t}\right){ }^{238} \mathrm{~Np},{ }^{238} \mathrm{U}\left({ }^{3} \mathrm{He},{ }^{4} \mathrm{He}\right){ }^{237} \mathrm{U}$ as surrogates for neutroninduced reactions on ${ }^{238} \mathrm{U},{ }^{237} \mathrm{~Np}$ and ${ }^{236} \mathrm{U}$, respectively, for which good quality data exist. The experimental set-up enabled the measurement of fission and gamma-decay probabilities. First results are presented and discussed.
\end{abstract}

\section{Introduction}

Neutron-induced cross sections of short-lived nuclei are crucial for fundamental nuclear physics, astrophysics and also for applications such as reactor physics. In particular, (n, $\gamma$ ) cross sections on minor actinides are one of the largest sources of uncertainty in modelling new reactors for nuclear waste transmutation using fast neutrons. However, very often the high radioactivity of the actinides samples makes the direct measurement of these cross sections extremely difficult. The surrogatereaction method is an indirect way of determining cross sections for compound-nuclear reactions. This method was first proposed by J. D. Cramer and H. C. Britt [1] in the seventies. It consists of using a transfer reaction to produce the same decaying nucleus as the one formed in the desired neutron-induced reaction. The transfer reaction leads to the heavy recoil nucleus of interest and to an ejectile. The identification of the ejectile permits to determine the mass $A$ and charge $Z$ of the decaying nucleus. In addition, one can deduce the excitation energy $E^{*}$ of the heavy nucleus by measuring the kinetic energy and the emission angle of the ejectile. The number of coincidences between the ejectiles and the decay products normalized to the total number of detected ejectiles 
allows for the extraction of the decay probability for the corresponding decay channel $P_{\text {decay }}^{A \text { exp }}\left(E^{*}\right)$.

According to the surrogate-reaction method, the neutron-induced cross section for the nucleus $A-1$ is then given by the equation:

$$
\sigma_{\text {decay }}^{A-1}\left(E_{n}\right) \cong \sigma_{C N}^{A}\left(E_{n}\right) P_{\text {decay }}^{A \text { exp }}\left(E_{n}\right)
$$

where $\sigma_{C N}^{A}\left(E_{n}\right)$ is the cross section for the formation of the compound nucleus $A$ after absorption of a neutron with energy $E_{n}$, it is usually obtained using the optical model. The incident neutron energy $E_{n}$ and the excitation energy $E^{*}$ of the compound nucleus $A$ are related by the expression $E^{*}=S_{n}+E_{n} \cdot(A-1) / A$, where $S_{n}$ is the one-neutron separation energy in the nucleus $A$. The benefit of the surrogate method is that in some cases the target needed is stable or less radioactive than the target of the corresponding neutron-induced reaction. Therefore, the surrogate-reaction method may enable neutron-induced cross sections to be extracted for nuclear reactions on short-lived nuclei that otherwise cannot be measured. For eq. (1) to apply two conditions have to be fulfilled: the decaying nucleus has to be a compound nucleus and the decay probability has to be independent of the spin $J$ and parity $\pi$ of the decaying nucleus. However, at low excitation energies the decay probability strongly depends on $J^{\pi}$. In addition, there may be important differences between the $J^{\pi}$ distributions populated in the neutron and transfer reactions. Therefore, considerable deviations between the neutron-induced results and the ones obtained with the surrogate method may exist. While it is rather well established that the surrogate method works well for fission at sufficiently high $E^{*}$ (see e.g. [2]), several recent experiments have shown that gamma decay is very sensitive to the differences in the populated $J^{\pi}$ distributions [3-5], which leads to significant discrepancies between the surrogate results and the neutron-induced data at the lowest excitation energies. This is probably due to the spin-parity selectivity of neutron emission [5]. This selectivity decreases strongly as the level density of the residual nucleus after neutron emission increases. Therefore, the discrepancies between the surrogate results and the neutron-induced data are expected to decrease with increasing mass of the decaying nucleus and with increasing excitation energy. In this work we study the validity of the surrogate method in the actinide region using an improved experimental set-up that enables the measurement of fission and gamma-decay probabilities. That allows us to investigate the two main issues determining the validity of the surrogate method: the "compound" character of the decaying nucleus and the $J^{\pi}$ dependence of the decay probabilities.

\section{Experiment}

The experiment was performed in June 2012 at the Oslo Cyclotron Laboratory (OCL). We used a ${ }^{238} \mathrm{U}$ target with $99.7 \%$ isotopic purity produced at GSI. Two different beams were used, a deuteron beam of $15 \mathrm{MeV}$ and a ${ }^{3} \mathrm{He}$ beam of $24 \mathrm{MeV}$. The experimental set-up is represented in Fig. 1. The ejectiles were detected at backward angles with the SiRi multi-strip silicon $\Delta \mathrm{E} / \mathrm{E}$ detector [6]. SiRi provided the identification of the ejectiles, as well as their kinetic energy and angle. Fission fragments were detected in coincidence with the ejectiles. The fission detector was located at forward angles and consisted of 4 PPACs covering a solid angle of $40 \%$ out of $4 \pi$. The reaction chamber housing SiRi, the PPACs and the ${ }^{238} \mathrm{U}$ target was surrounded by the CACTUS array with 26 high-efficiency NaI detectors. CACTUS was used to detect gamma rays with energies ranging from about $200 \mathrm{keV}$ to $12 \mathrm{MeV}$ in coincidence with the ejectiles.

The different transfer reactions on the ${ }^{238} \mathrm{U}$ target lead to the production of various heavy residues. Table 1 lists the transfer channels considered in the present work, and the corresponding neutroninduced reactions to which the surrogate results can be compared. 


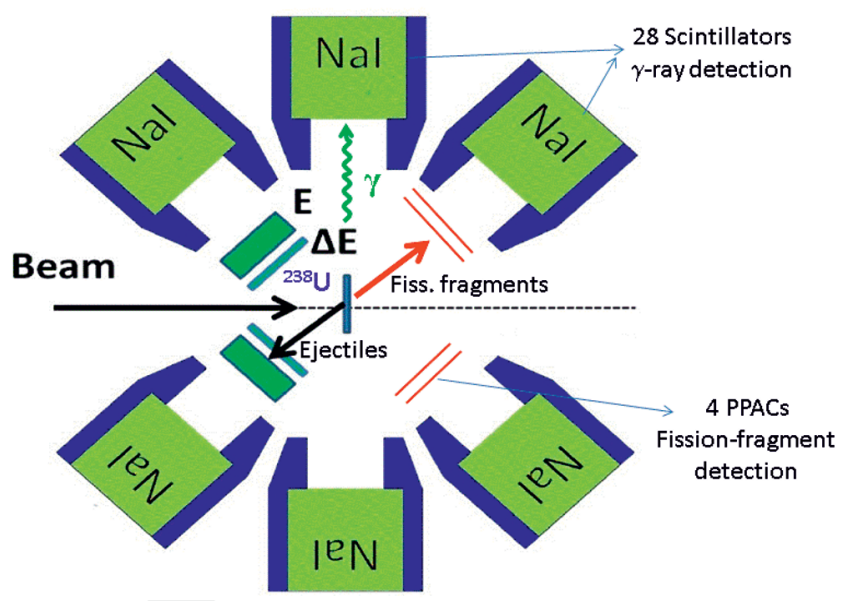

Fig. 1. Schematic view of the set-up for decay-probability measurements with the SIRI telescope and the fission detector inside the CACTUS NaI array.

Table 1. Transfer reactions investigated in this work and the corresponding neutron-induced fission reactions.

\begin{tabular}{|c|c|}
\hline Transfer reactions & Neutron-induced reactions \\
\hline${ }^{238} \mathrm{U}(\mathrm{d}, \mathrm{p}){ }^{239} \mathrm{U}^{*}$ & ${ }^{238} \mathrm{U}(\mathrm{n}, \mathrm{f})$ and ${ }^{238} \mathrm{U}(\mathrm{n}, \gamma)$ \\
\hline${ }^{238} \mathrm{U}\left({ }^{3} \mathrm{He}, \mathrm{t}\right)^{238} \mathrm{~Np} *$ & ${ }^{237} \mathrm{~Np}(\mathrm{n}, \mathrm{f})$ and ${ }^{237} \mathrm{~Np}(\mathrm{n}, \gamma)$ \\
\hline${ }^{238} \mathrm{U}\left({ }^{3} \mathrm{He},{ }^{4} \mathrm{He}\right){ }^{237} \mathrm{U}^{*}$ & ${ }^{236} \mathrm{U}(\mathrm{n}, \mathrm{f})$ and ${ }^{236} \mathrm{U}(\mathrm{n}, \gamma)$ \\
\hline
\end{tabular}

Fission or gamma-decay probabilities are obtained from the following the expression:

$$
P_{\text {decay }}^{\exp }\left(E^{*}\right)=\frac{N_{\text {coin }}\left(E^{*}\right)}{N_{\text {singles }}\left(E^{*}\right) \varepsilon_{\text {decay }}\left(E^{*}\right)}
$$

where $N_{\text {coin }}$ is the number of coincidences between the ejectiles and the fission or the gamma detector, $N_{\text {singles }}$ is the total number of detected ejectiles and $\varepsilon_{\text {decay }}$ is the efficiency of the fission or the gamma detector.

\section{Preliminary results}

The results presented in this section have been obtained from the data analysis for one telescope strip out of 64 . The selected strip was located at 126 degrees relative to the beam. Because of the relatively low statistics, these results must be considered as preliminary. The left part of Fig. 2 shows the fission probability of ${ }^{239} \mathrm{U}^{*}$ as a function of excitation energy obtained from the ${ }^{238} \mathrm{U}(\mathrm{d}, \mathrm{p})$ reaction in comparison with the neutron-induced fission probability of ${ }^{239} \mathrm{U}^{*}$ as given by TALYS [7]. For $E^{*}>6 \mathrm{MeV}$ the agreement between the two curves is very good. However, above $6.5 \mathrm{MeV}$ the results from the surrogate reaction are clearly below the neutron-induced results. The difference amounts to $25 \%$ at most. The reason for this discrepancy could be the breakup of the deuteron, which means that the neutron that is transferred in the stripping $(\mathrm{d}, \mathrm{p})$ reaction to the ${ }^{238} \mathrm{U}$ target 
nucleus escapes into the breakup phase space before the nucleus ${ }^{239} \mathrm{U}$ becomes a compound nucleus. This leads to a background of "sterile" protons that contaminates the singles proton spectrum. This phenomenon was already observed by Britt and Cramer [8] and a theoretical investigation was initiated [9]. It is interesting to note that the fission probability obtained by Britt and Cramer for the ${ }^{238} \mathrm{U}(\mathrm{d}, \mathrm{p})$ reaction is about two times smaller than the one obtained in this work. This could be due to the higher energy of the deuteron beam, $18 \mathrm{MeV}$, used in their experiment. To understand these results model calculations based on the theory of partial fusion are necessary.
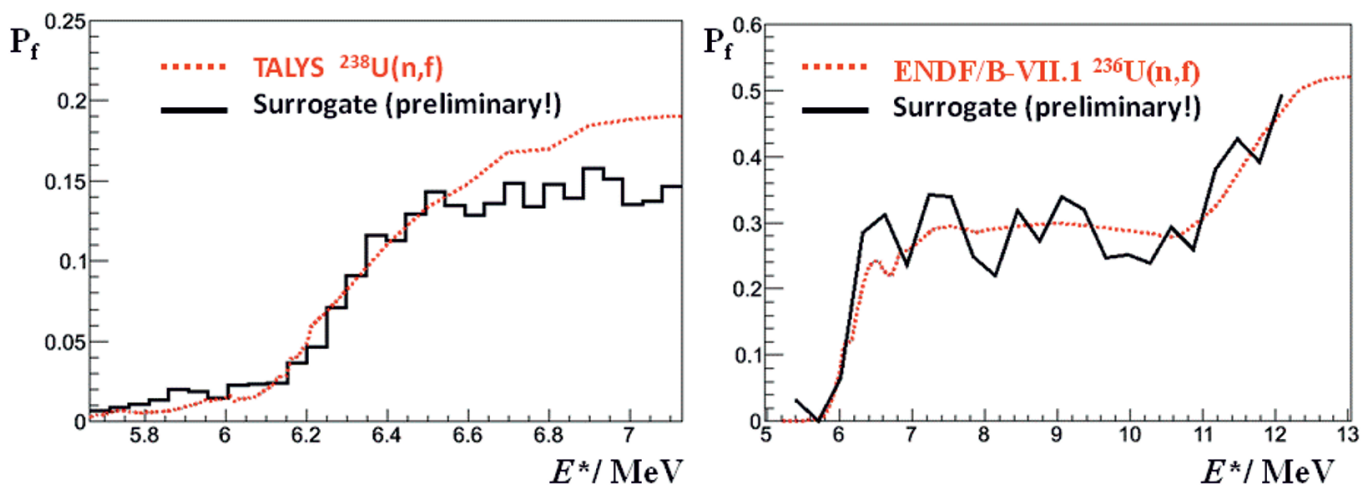

Fig. 2. Preliminary results for the fission probabilities of ${ }^{239} U^{*}$ (left) and ${ }^{237} U^{*}$ (right) obtained in the ${ }^{238} U(\mathrm{~d}, p$ ) and in the ${ }^{238} \mathrm{U}\left({ }^{3} \mathrm{He},{ }^{4} \mathrm{He}\right)$ reactions, respectively, compared to TALYS [7] and ENDF/B-VII.1.

On the right of Fig. 2, the results for the fission probability of ${ }^{237} \mathrm{U}^{*}$ induced in the ${ }^{238} \mathrm{U}\left({ }^{3} \mathrm{He},{ }^{4} \mathrm{He}\right)$ reaction are presented. The large fluctuations of the surrogate result are purely statistical due to the limited number of events analysed. Aside from the statistical fluctuations, there is an overall good agreement between the surrogate and the neutron-induced data represented by the ENDF/B-VII.1 evaluation. Since the target and the experimental set-up used to measure the fission probability of ${ }^{239} \mathrm{U}^{*}$ and ${ }^{237} \mathrm{U}^{*}$ were the same, there is no experimental systematic error that explains the lowering of the fission probability of ${ }^{239} \mathrm{U}^{*}$ for $E^{*}>6.5 \mathrm{MeV}$.

Fig. 3 represents the ratio $\mathrm{N}_{\gamma \text {-coin }} / \mathrm{N}_{\text {singles }}$, i.e. the number of coincidences between a proton and 1 $\mathrm{NaI}$ divided by the total number of detected protons, for the ${ }^{238} \mathrm{U}(\mathrm{d}, \mathrm{p})$ reaction. This quantity divided by the cascade-detection efficiency of CACTUS gives the gamma-decay probability, see eq. (2). As expected, we can see that the ratio decreases very rapidly at an $E^{*}$ of about $4.9 \mathrm{MeV}$, in very good agreement with the $S_{\mathrm{n}}$ of ${ }^{239} \mathrm{U}^{*}$. This decrease is due to the onset of neutron evaporation that competes with gamma emission and becomes the dominant de-excitation mode several hundreds of $\mathrm{keV}$ after $S_{\mathrm{n}}$. Gamma-rays emitted following neutron evaporation were removed from $\mathrm{N}_{\gamma \text {-coin }}$ by applying a threshold to the energy of the NaI detectors. As can be seen on the left of Fig. 2, fission sets in at about $5.7 \mathrm{MeV}$. Therefore, up to this excitation energy, the gamma-decay probability of ${ }^{239} \mathrm{U}^{*}$ is not affected by the background coming from gamma emission by the fission fragments.

\section{Conclusions and perspectives}

We performed an experiment at the OCL to further investigate the validity of the surrogate method in the actinide region. The first results for the fission probability of ${ }^{239} \mathrm{U}^{*}$ induced in the ${ }^{238} \mathrm{U}(\mathrm{d}, \mathrm{pf})$ reaction show a systematic lowering that could be due to deuteron breakup. These results will provide very valuable information on the process of formation of a compound nucleus after the stripping $(d, p)$ reaction. Note that the $(d, p)$ reaction is particularly interesting to simulate neutroninduced reactions in inverse kinematics with radioactive beams. 


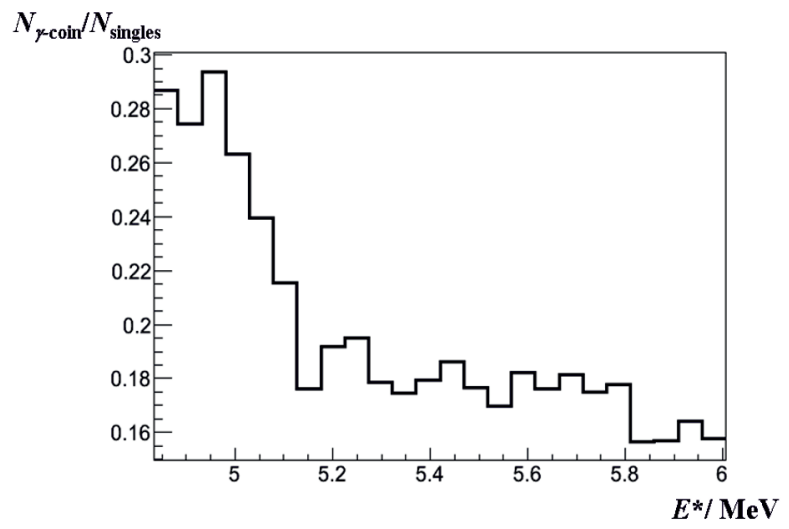

Fig. 3. Preliminary results for the number of coincidences between protons and $1 \mathrm{NaI}$ divided by the number of detected protons for the reaction ${ }^{238} \mathrm{U}(\mathrm{d}, \mathrm{p} \gamma){ }^{239} \mathrm{U}^{*}$.

In spite of the large statistical fluctuations, we observe that the fission probability of ${ }^{237} \mathrm{U}^{*}$ induced in the ${ }^{236} \mathrm{U}\left({ }^{3} \mathrm{He},{ }^{4} \mathrm{He}\right)$ reaction is in overall good agreement with the neutron-induced results. This means that the lowering of the fission probability found for the ${ }^{238} \mathrm{U}(\mathrm{d}, \mathrm{pf})$ reaction is not due to a systematic error in the experimental setup. The ratio $N_{\gamma \text {-coin }} / N_{\text {singles }}$ was extracted for the ${ }^{238} U(d, p \gamma)$ reaction. It presents the expected step decrease at the neutron separation energy of ${ }^{239} \mathrm{U}^{*}$ that reflects the competition between gamma emission and neutron evaporation. To obtain the gamma-decay probability, this ratio has to be corrected for the cascade-detection efficiency of CACTUS. The latter will be obtained from a detailed analysis of the experimental data. The complete analysis of the data will allow us to determine also the fission and gamma-decay probabilities associated to the reactions ${ }^{238} \mathrm{U}\left({ }^{3} \mathrm{He},{ }^{4} \mathrm{He}\right),{ }^{238} \mathrm{U}\left({ }^{3} \mathrm{He}, \mathrm{t}\right)$. The ensemble of the results from this work will provide an important step forward for establishing to which extent the surrogate method can be applied to extract neutroninduced cross sections in the actinide region.

\section{Acknowledgements}

We would like to thank the staff of the OCL for the support during the experiment, Bettina Lommel and the GSI Target Laboratory for the production of high-quality ${ }^{238} \mathrm{U}$ targets and Pascal Romain for performing the TALYS calculation. This work was supported by the European Commission within the $7^{\text {th }}$ Framework Programme through Fission-2010-ERINDA (Project No 269499), by the European Atomic Energy Community's 7th Framework Programme under grant agreement $n^{\circ}$ FP7249671 (ANDES) and by the French national research programme GEDEPEON. MW acknowledges support from the National Research Foundation of South Africa.

\section{References}

1. J. D. Cramer and H. C. Britt, Nucl. Sci. Eng. 41, 177 (1970)

2. G. Kessedjian et al., Phys. Lett. B 692, 297 (2010)

3. N. D. Scielzo et al., Phys. Rev. C 81, 034608 (2010)

4. J. N. Wilson et al., Phys. Rev. C 85, 034607 (2012)

5. G. Boutoux et al., Phys. Lett. B 712, 319 (2012)

6. M. Guttormsen et al., Nucl. Istrum. Meth. A 648, 168 (2011)

7. A. J. Koning et al., User Manual TALYS, www.talys.eu

8. H. C. Britt and J. D. Cramer, Phys. Rev. C 2, 1758 (1970)

9. J. E. Escher, Rev. Mod. Phys. 84, 353 (2012) 